\title{
Fermionic model of unitary transport of qubits from a black hole
}

\author{
Bogusław Broda $\odot^{*}$ \\ Department of Theoretical Physics, Faculty of Physics and Applied Informatics, \\ University of Łódź, Pomorska 149/153, 90-236 Łódź, Poland
}

(Received 22 July 2020; accepted 11 December 2020; published 25 January 2021)

\begin{abstract}
Inspired by a recent model of Osuga and Page, we propose an explicitly unitary fermionic toy model for transferring information from a black hole to the outgoing radiation. The model treats the unitary evolution as a composition of the Hawking pair creation outside the black hole and of pair annihilation inside the black hole.
\end{abstract}

DOI: $10.1103 /$ PhysRevD.103.025022

\section{INTRODUCTION}

The black hole (BH) information (loss) paradox concerns difficulties around the issue of unitarity of $\mathrm{BH}$ evaporation (for recent reviews see e.g., [1-4]). There are a lot of approaches proposed to date to analyze and resolve the paradox-some of them suggest to study simplified situations embodied in various qubit models (see e.g., [5-12]). A successful model of BH evolution should include a description of particle pair production according to the Hawking prescription, following gradual evaporation ("vanishing") of the $\mathrm{BH}$, and it should be unitary. A model strictly motivated by actual physical phenomena would certainly be greatly appreciated, but in fact any model respecting at least general physical laws, even without any real physical mechanism built in, would be welcome as a "proof of concept."

Recently Osuga and Page [12] (inspired by [13]) have proposed an explicitly unitary toy qubit transport model for $\mathrm{BH}$ evaporation (without firewalls). Another version of the model (with additional features) has been presented in [14]. In the present paper, building on both models, we propose yet another toy qubit transport model for $\mathrm{BH}$ evaporation, which is explicitly unitary. Since the model, by assumption, operates on qubits and the particle pair production scheme exactly follows the Hawking mechanism for fermions, we shall work in terms of fermionic modes rather than bosonic ones. A new and important feature of our present proposal is explicit incorporation of the (fermionic) Hawking pair creation mechanism into the chain of unitary processes. In other words, the global unitary evolution considered is

\footnotetext{
*boguslaw.broda@uni.lodz.pl
}

Published by the American Physical Society under the terms of the Creative Commons Attribution 4.0 International license. Further distribution of this work must maintain attribution to the author(s) and the published article's title, journal citation, and DOI. Funded by SCOAP . given by the composition $U=U^{\prime \prime} \cdot U^{\prime}$, where $U^{\prime}$ corresponds to creation of fermionic pairs outside a $\mathrm{BH}$ according to the Hawking prescription, whereas $U^{\prime \prime}$ corresponds to annihilation of fermion pairs inside the $\mathrm{BH}$ (as described in [14]).

For the reader's convenience, we will follow the notation of [12] (and [14]) as closely as possible.

\section{THE TOY MODEL}

An initial total quantum state describing a newly formed (fermionic) BH and "fermionic radiation" in the vacuum state is assumed in the following (partially product) form [14] (cf. [12]):

$$
|\Psi\rangle=\sum_{q_{1}, q_{2}, \ldots, q_{N}=0}^{1} A_{q_{1} q_{2} \cdots q_{N}} \bigotimes_{k=1}^{N}\left|q_{k}\right\rangle_{a_{k}} \otimes|\mathrm{O}\rangle_{b_{k} c_{k}} .
$$

Here $A_{q_{1} q_{2} \cdots q_{N}}$ are amplitudes for inner BH modes $a_{k}$, which encode a quantum state of the $\mathrm{BH}$, and the vacuum state for fermionic radiation is

$$
|\mathrm{O}\rangle_{b_{k} c_{k}} \equiv|0\rangle_{b_{k}} \otimes|0\rangle_{c_{k}}
$$

where the Hawking (fermionic) modes $b_{k}$ and $c_{k}$ are infalling and outgoing modes, respectively. The same range of indices $(k=1,2, \ldots, N)$ postulated for $\mathrm{BH}$ modes $a_{k}$ and $b_{k}, c_{k}$ pairs is not only a convenient computational simplification in our model but also it is a physically justified assumption, at least approximately (see e.g., [14]).

In the language of $k$-mode blocks, the first step of (unitary) evolution denoted by $U_{k}^{\prime}$ yields the Hawking (fermion) pair for a single " $k$ " mode, i.e.,

$$
U_{k}^{\prime}\left(\left|q_{k}\right\rangle_{a_{k}} \otimes|\mathrm{O}\rangle_{b_{k} c_{k}}\right)=\left|q_{k}\right\rangle_{a_{k}} \otimes\left|\mathrm{H}_{1}\right\rangle_{b_{k} c_{k}},
$$

where the fermionic Hawking state can be chosen in the form [cf. Eq. (116) in [15]] 
$\left|\mathrm{H}_{1}\right\rangle_{b_{k} c_{k}} \equiv \cos \omega_{k}|0\rangle_{b_{k}} \otimes|0\rangle_{c_{k}}+\sin \omega_{k}|1\rangle_{b_{k}} \otimes|1\rangle_{c_{k}}$,

with $\omega_{k}$ determined by $\mathrm{BH}$ parameter(s).

The total (i.e., for all modes $k$ ) $U^{\prime}$-evolution yields by virtue of (3) the total (intermediate) state

$$
\left|\Psi^{\prime}\right\rangle=\sum_{q_{1}, q_{2}, \ldots, q_{N}=0}^{1} A_{q_{1} q_{2} \cdots q_{N}} \otimes_{i=1}^{N}\left|q_{k}\right\rangle_{a_{k}} \otimes\left|\mathrm{H}_{1}\right\rangle_{b_{k} c_{k}} .
$$

We could possibly consider a slight generalization of the unitary evolution (3) allowing some unitary transformation $\mathcal{U}_{k}^{\prime}\left|q_{k}\right\rangle_{a_{k}}$ of the internal BH mode $a_{k}$ on the rhs of (3), but we ignore this option, because it would merely give rise to a redefinition of $A$ amplitudes $\left(A_{q_{1} q_{2} \cdots q_{N}} \mapsto A_{q_{1} q_{2} \cdots q_{N}}^{\prime}\right)$ in the final state.

The second step of (unitary) evolution denoted by $U_{k}^{\prime \prime}$ yields particle pair annihilation inside the $\mathrm{BH}$ [see [14] and cf. Eq. (3.3) in $[12,13]]$, i.e.,

$$
U_{k}^{\prime \prime}\left(\left|q_{k}\right\rangle_{a_{k}} \otimes\left|\mathrm{H}_{1}\right\rangle_{b_{k} c_{k}}\right)=|\mathrm{O}\rangle_{a_{k} b_{k}} \otimes\left|q_{k}\right\rangle_{c_{k}},
$$

where the vacuum state $|\mathrm{O}\rangle_{a_{k} b_{k}}$ is defined analogously to (2) (with appropriate replacements of modes). have

Consequently, for the entire evolution $U_{k} \equiv U_{k}^{\prime \prime} \cdot U_{k}^{\prime}$ we

$$
U_{k}\left(\left|q_{k}\right\rangle_{a_{k}} \otimes|\mathrm{O}\rangle_{b_{k} c_{k}}\right)=|\mathrm{O}\rangle_{a_{k} b_{k}} \otimes\left|q_{k}\right\rangle_{c_{k}}
$$

and the final total state assumes the form

$$
\left|\Psi^{\prime \prime}\right\rangle=\sum_{q_{1}, q_{2}, \ldots, q_{N}=0}^{1} A_{q_{1} q_{2} \cdots q_{N}} \stackrel{N}{\otimes}|\mathrm{O}\rangle_{a_{k} b_{k}} \otimes\left|q_{k}\right\rangle_{c_{k}} .
$$

Equation (8) means that all information has been transferred from a $\mathrm{BH}$ to the outgoing radiation, and the $\mathrm{BH}$ is in the vacuum state.

Obviously, the total operators $U^{\prime}, U^{\prime \prime}, U$ are the following tensor products of the above-defined $k$-mode operators,

$$
U^{\prime}=\bigotimes_{k=1}^{N} U_{k}^{\prime}, \quad U^{\prime \prime}=\bigotimes_{k=1}^{N} U_{k}^{\prime \prime}, \quad U=\bigotimes_{k=1}^{N} U_{k}^{\prime \prime} \cdot U_{k}^{\prime} \equiv \bigotimes_{k=1}^{N} U_{k},
$$

respectively, and

$$
\begin{aligned}
U^{\prime}|\Psi\rangle & =\left|\Psi^{\prime}\right\rangle, \quad U^{\prime \prime}\left|\Psi^{\prime}\right\rangle=\left|\Psi^{\prime \prime}\right\rangle, \\
U|\Psi\rangle & \equiv U^{\prime \prime} \cdot U^{\prime}|\Psi\rangle=\left|\Psi^{\prime \prime}\right\rangle .
\end{aligned}
$$

\section{UNITARY OPERATORS}

We shall now explicitly derive the implicitly defined unitary operators $U_{k}^{\prime}, U_{k}^{\prime \prime}$ and $U_{k}$. To this end let us first observe the following elementary fact from linear algebra: namely, for each pair of orthonormal bases $\left\{\left|E_{\Lambda}\right\rangle\right\},\left\{\left|E_{\Lambda}^{\prime}\right\rangle\right\}$ $\left(\left\langle E_{\Lambda} \mid E_{\Lambda^{\prime}}\right\rangle=\left\langle E_{\Lambda}^{\prime} \mid E_{\Lambda^{\prime}}^{\prime}\right\rangle=\delta_{\Lambda \Lambda^{\prime}}\right)$ in a finite dimensional Hilbert space $\mathcal{H}$ we can construct an operator

$$
U=\sum_{\Lambda}\left|E_{\Lambda}^{\prime}\right\rangle\left\langle E_{\Lambda}\right|,
$$

which is explicitly unitary. Really, we can easily check that e.g.,

$U^{\dagger} \cdot U=\sum_{\Lambda, \Lambda^{\prime}}\left|E_{\Lambda}\right\rangle\left\langle E_{\Lambda}^{\prime} \mid E_{\Lambda^{\prime}}^{\prime}\right\rangle\left\langle E_{\Lambda^{\prime}}\left|=\sum_{\Lambda, \Lambda^{\prime}} \delta_{\Lambda \Lambda^{\prime}}\right| E_{\Lambda}\right\rangle\left\langle E_{\Lambda^{\prime}}\right|=\mathbb{I}$.

Since the total Hilbert space $\mathcal{H}$ is a tensor product of $N$ $k$-mode Hilbert spaces $\mathcal{H}_{k}$, i.e., $\mathcal{H}=\bigotimes_{k=1}^{N} \mathcal{H}_{k}$, we can confine our construction to the single $k$-mode space $\mathcal{H}_{k}=\mathcal{H}_{a_{k}} \otimes \mathcal{H}_{b_{k}} \otimes \mathcal{H}_{c_{k}}$, where $\operatorname{dim}_{\mathbb{C}} \mathcal{H}_{k}=2 \cdot 2 \cdot 2=8$. Then, our unitary operators will be defined by three eight-dimensional orthonormal ( $k$-dependent) bases in $\mathcal{H}_{k}$.

The first base, $\left\{\left|E_{\Lambda}\right\rangle_{k}\right\}_{\Lambda=0}^{7}$, assumes the following standard form:

$$
\begin{aligned}
\left|E_{0}\right\rangle_{k} & =|0\rangle_{a_{k}} \otimes|0\rangle_{b_{k}} \otimes|0\rangle_{c_{k}} \\
\left|E_{1}\right\rangle_{k} & =|0\rangle_{a_{k}} \otimes|0\rangle_{b_{k}} \otimes|1\rangle_{c_{k}} \\
\vdots & \\
\left|E_{7}\right\rangle_{k} & =|1\rangle_{a_{k}} \otimes|1\rangle_{b_{k}} \otimes|1\rangle_{c_{k}}
\end{aligned}
$$

(or $\left|E_{\Lambda}\right\rangle_{k}=|\Lambda\rangle_{a_{k} b_{k} c_{k}}$, in short).

The second ( $\tau$-dependent) base, $\left\{\left|E_{\Lambda}^{\prime}(\tau)\right\rangle_{k}\right\}_{\Lambda=0}^{7}$, is given by (for further convenience, we have also included expansions in terms of $\tau$ )

$$
\begin{aligned}
\left|E_{0}^{\prime}(\tau)\right\rangle_{k}= & |0\rangle_{a_{k}} \otimes\left[\cos \left(\omega_{k} \tau\right)|0\rangle_{b_{k}} \otimes|0\rangle_{c_{k}}\right. \\
& \left.+\sin \left(\omega_{k} \tau\right)|1\rangle_{b_{k}} \otimes|1\rangle_{c_{k}}\right] \\
\equiv & |0\rangle_{a_{k}} \otimes\left|\mathrm{H}_{\tau}\right\rangle_{b_{k} c_{k}} \\
\equiv & \cos \left(\omega_{k} \tau\right)\left|E_{0}\right\rangle_{k}+\sin \left(\omega_{k} \tau\right)\left|E_{3}\right\rangle_{k} \\
= & \left|E_{0}\right\rangle_{k}+\omega_{k} \tau\left|E_{3}\right\rangle_{k}+\mathcal{O}\left(\tau^{2}\right) \\
\left|E_{1}^{\prime}\right\rangle_{k}= & |0\rangle_{a_{k}} \otimes|0\rangle_{b_{k}} \otimes|1\rangle_{c_{k}} \equiv\left|E_{1}\right\rangle_{k} \\
\left|E_{2}^{\prime}\right\rangle_{k}= & |0\rangle_{a_{k}} \otimes|1\rangle_{b_{k}} \otimes|0\rangle_{c_{k}} \equiv\left|E_{2}\right\rangle_{k} \\
\left|E_{3}^{\prime}(\tau)\right\rangle_{k}= & |0\rangle_{a_{k}} \otimes\left[-\sin \left(\omega_{k} \tau\right)|0\rangle_{b_{k}} \otimes|0\rangle_{c_{k}}\right. \\
& \left.+\cos \left(\omega_{k} \tau\right)|1\rangle_{b_{k}} \otimes|1\rangle_{c_{i}}\right] \\
\equiv & |0\rangle_{a_{k}} \otimes\left|\mathrm{H}_{\tau}^{\perp}\right\rangle_{b_{k} c_{k}} \\
\equiv & \cos \left(\omega_{k} \tau\right)\left|E_{3}\right\rangle_{k}-\sin \left(\omega_{k} \tau\right)\left|E_{0}\right\rangle_{k} \\
= & \left|E_{3}\right\rangle_{k}-\omega_{k} \tau\left|E_{0}\right\rangle_{k}+\mathcal{O}\left(\tau^{2}\right) \\
\left|E_{4}^{\prime}(\tau)\right\rangle_{k}= & |1\rangle_{a_{k}} \otimes\left[\cos \left(\omega_{k} \tau\right)|0\rangle_{b_{k}} \otimes|0\rangle_{c_{k}}\right. \\
& \left.+\sin \left(\omega_{k} \tau\right)|1\rangle_{b_{k}} \otimes|1\rangle_{c_{k}}\right] \\
\equiv & |1\rangle_{a_{k}} \otimes\left|\mathrm{H}_{\tau}\right\rangle_{b_{k} c_{k}}
\end{aligned}
$$




$$
\begin{aligned}
& \equiv \cos \left(\omega_{k} \tau\right)\left|E_{4}\right\rangle_{k}+\sin \left(\omega_{k} \tau\right)\left|E_{7}\right\rangle_{k} \\
&=\left|E_{4}\right\rangle_{k}+\omega_{k} \tau\left|E_{7}\right\rangle_{k}+\mathcal{O}\left(\tau^{2}\right) \\
&\left|E_{5}^{\prime}\right\rangle_{k}=|0\rangle_{a_{k}} \otimes|0\rangle_{b_{k}} \otimes|1\rangle_{c_{k}} \equiv\left|E_{5}\right\rangle_{k} \\
&\left|E_{6}^{\prime}\right\rangle_{k}=|0\rangle_{a_{k}} \otimes|0\rangle_{b_{k}} \otimes|1\rangle_{c_{k}} \equiv\left|E_{6}\right\rangle_{k} \\
&\left|E_{7}^{\prime}(\tau)\right\rangle_{k}=|1\rangle_{a_{k}} \otimes\left[-\sin \left(\omega_{k} \tau\right)|0\rangle_{b_{k}} \otimes|0\rangle_{c_{k}}\right. \\
&\left.+\cos \left(\omega_{k} \tau\right)|1\rangle_{b_{k}} \otimes|1\rangle_{c_{k}}\right] \\
& \equiv|1\rangle_{a_{k}} \otimes\left|\mathrm{H}_{\tau}^{\perp}\right\rangle_{b_{k} c_{k}} \\
& \equiv \cos \left(\omega_{k} \tau\right)\left|E_{7}\right\rangle_{k}-\sin \left(\omega_{k} \tau\right)\left|E_{4}\right\rangle_{k} \\
&=\left|E_{7}\right\rangle_{k}-\omega_{k} \tau\left|E_{4}\right\rangle_{k}+\mathcal{O}\left(\tau^{2}\right),
\end{aligned}
$$

where $\left|\mathrm{H}_{\tau}^{\perp}\right\rangle$ denotes a Hawking state orthogonal to the Hawking state $\left|\mathrm{H}_{\tau}\right\rangle$. Since, from a geometrical point of view, $\omega_{k}$ is an angle of rotation in the eight-dimensional Hilbert space $\mathcal{H}_{k}$ for the $k$-mode sector, the auxiliary "time" parameter $\tau$ is dimensionless. More precisely, $\tau$ is a scale parameter governing the evolution, and $\tau \in[0,1]$. In particular, for $\tau=1$ the evolution is supposed to be completed.

The third base, $\left\{\left|E_{\Lambda}^{\prime \prime}(\tau)\right\rangle_{k}\right\}_{\Lambda=0}^{7}$, is given by

$$
\begin{aligned}
\left|E_{0}^{\prime \prime}\right\rangle_{k}= & |0\rangle_{a_{k}} \otimes|0\rangle_{b_{k}} \otimes|0\rangle_{c_{k}} \equiv\left|E_{0}\right\rangle_{k} \\
\left|E_{1}^{\prime \prime}(\tau)\right\rangle_{k}= & \cos \left(\frac{\pi \tau}{2}\right)|0\rangle_{a_{k}} \otimes|0\rangle_{b_{k}} \otimes|1\rangle_{c_{k}}-\sin \left(\frac{\pi \tau}{2}\right)|1\rangle_{a_{k}} \\
& \otimes|0\rangle_{b_{k}} \otimes|0\rangle_{c_{k}} \\
& \equiv \cos \left(\frac{\pi \tau}{2}\right)\left|E_{1}\right\rangle_{k}-\sin \left(\frac{\pi \tau}{2}\right)\left|E_{4}\right\rangle_{k} \\
= & \left|E_{1}\right\rangle_{k}-\frac{\pi \tau}{2}\left|E_{4}\right\rangle_{k}+\mathcal{O}\left(\tau^{2}\right) \\
\left|E_{2}^{\prime \prime}\right\rangle_{k}= & |0\rangle_{a_{k}} \otimes|1\rangle_{b_{k}} \otimes|0\rangle_{c_{k}} \equiv\left|E_{2}\right\rangle_{k} \\
\left|E_{3}^{\prime \prime}\right\rangle_{k}= & |0\rangle_{a_{k}} \otimes|1\rangle_{b_{k}} \otimes|1\rangle_{c_{k}} \equiv\left|E_{3}\right\rangle_{k} \\
\left|E_{4}^{\prime \prime}(\tau)\right\rangle_{k}= & \sin \left(\frac{\pi \tau}{2}\right)|0\rangle_{a_{k}} \otimes|0\rangle_{b_{k}} \otimes|1\rangle_{c_{k}}+\cos \left(\frac{\pi \tau}{2}\right)|1\rangle_{a_{k}} \\
& \otimes|0\rangle_{b_{k}} \otimes|0\rangle_{c_{k}} \\
\equiv & \cos \left(\frac{\pi \tau}{2}\right)\left|E_{4}\right\rangle_{k}+\sin \left(\frac{\pi \tau}{2}\right)\left|E_{1}\right\rangle_{k} \\
= & \left|E_{4}\right\rangle_{k}+\frac{\pi \tau}{2}\left|E_{1}\right\rangle_{k}+\mathcal{O}\left(\tau^{2}\right) \\
\left|E_{5}^{\prime \prime}\right\rangle_{k}= & |1\rangle_{a_{k}} \otimes|0\rangle_{b_{k}} \otimes|1\rangle_{c_{k}} \equiv\left|E_{5}\right\rangle_{k} \\
\left|E_{6}^{\prime \prime}\right\rangle_{k}= & |1\rangle_{a_{k}} \otimes|1\rangle_{b_{k}} \otimes|0\rangle_{c_{k}} \equiv\left|E_{6}\right\rangle_{k} \\
\left|E_{7}^{\prime \prime}\right\rangle_{k}= & |1\rangle_{a_{k}} \otimes|1\rangle_{b_{k}} \otimes|1\rangle_{c_{k}} \equiv\left|E_{7}\right\rangle_{k} .
\end{aligned}
$$

Now we define ( $\tau$-dependent) unitary operators according to the recipe (11) as follows:

$$
\begin{gathered}
U_{k}^{\prime}(\tau)=\sum_{\Lambda=0}^{7}\left|E_{\Lambda}^{\prime}(\tau)\right\rangle_{k}\left\langle\left. E_{\Lambda}\right|_{k},\right. \\
U_{k}^{\prime \prime}(\tau)=\sum_{\Lambda=0}^{7}\left|E_{\Lambda}^{\prime \prime}(\tau)\right\rangle_{k}\left\langle\left. E_{\Lambda}^{\prime}(\tau)\right|_{k},\right.
\end{gathered}
$$

and

$$
\begin{aligned}
U_{k}(\tau) & \equiv U_{k}^{\prime \prime}(\tau) \cdot U_{k}^{\prime}(\tau)=\sum_{\Lambda, \Lambda^{\prime}=0}^{7}\left|E_{\Lambda}^{\prime \prime}(\tau)\right\rangle\left\langle E_{\Lambda}^{\prime}(\tau) \mid E_{\Lambda^{\prime}}^{\prime}(\tau)\right\rangle\left\langle E_{\Lambda^{\prime}}\right| \\
& =\sum_{\Lambda=0}^{7}\left|E_{\Lambda}^{\prime \prime}(\tau)\right\rangle\left\langle E_{\Lambda}\right|
\end{aligned}
$$

We can easily confirm that for $\tau=1$ the (explicitly) unitary operators (16), (17) and (18) act according to the rules (3), (6) and (7), respectively. For example, for $U_{k}^{\prime}\left(\equiv U_{k}^{\prime}(1)\right)$ we confirm that

$$
\begin{aligned}
& U_{k}^{\prime}\left(\left|q_{k}\right\rangle_{a_{k}} \otimes|\mathrm{O}\rangle_{b_{k} c_{k}}\right) \\
& =U_{k}^{\prime}\left\{\left[\left(1-q_{k}\right)|0\rangle_{a_{k}}+q_{k}|1\rangle_{a_{k}}\right] \otimes|\mathrm{O}\rangle_{b_{k} c_{k}}\right\} \\
& =\sum_{\Lambda=0}^{7}\left|E_{\Lambda}^{\prime}(1)\right\rangle_{k}\left\langle\left. E_{\Lambda}\right|_{k}\left[\left(1-q_{k}\right)\left|E_{0}\right\rangle_{k}+q_{k}\left|E_{4}\right\rangle_{k}\right]\right. \\
& =\left(1-q_{k}\right)\left|E_{0}^{\prime}(1)\right\rangle_{k}+q_{k}\left|E_{4}^{\prime}(1)\right\rangle_{k}=\left[\left(1-q_{k}\right)|0\rangle_{a_{k}}\right. \\
& \left.\quad+q_{k}|1\rangle_{a_{k}}\right] \otimes\left|\mathrm{H}_{1}\right\rangle_{b_{k} c_{k}} \\
& =\left|q_{k}\right\rangle_{a_{k}} \otimes\left|\mathrm{H}_{1}\right\rangle_{b_{k} c_{k}},
\end{aligned}
$$

as expected [see (3)].

\section{PHYSICAL PICTURE}

Now, let us determine a corresponding (infinitesimal) generator $\mathbb{U}_{k}$ ("Hamiltonian") for the unitary evolution operator $U_{k}(\tau)$, i.e.,

$$
U_{k}(\tau)=\mathbb{I}_{k}-i \tau \llbracket \mathbb{\boxplus}_{k}+\mathcal{O}\left(\tau^{2}\right) .
$$

To this end we will utilize expansions (in terms of the time parameter $\tau$ ) of the bases $\left\{\left|E_{\Lambda}^{\prime}(\tau)\right\rangle\right\}$ and $\left\{\left|E_{\Lambda}^{\prime \prime}(\tau)\right\rangle\right\}$ given in (14) and (15), respectively, around the standard base $\left\{\left|E_{\Lambda}\right\rangle\right\}$.

To make a comparison to a known case (i.e., to the fermion squeezing operator [16,17]), let us derive the form of the generator $\mathbb{U}_{k}^{\prime}$ for the unitary transformation $U_{k}^{\prime}(\tau)$ (16). By virtue of (16), (14) and (20) (with unprimed quantities replaced by primed ones) we get

$$
\begin{aligned}
U_{k}^{\prime}(\tau)= & \mathbb{I}_{k}-i \tau i \omega_{k}\left(| E _ { 3 } \rangle _ { k } \langle E _ { 0 } | _ { k } - | E _ { 0 } \rangle _ { k } \left\langle\left.E_{3}\right|_{k}\right.\right. \\
& +\left|E_{7}\right\rangle_{k}\left\langle\left. E_{4}\right|_{k}-\mid E_{4}\right\rangle_{k}\left\langle\left. E_{7}\right|_{k}\right)+\mathcal{O}\left(\tau^{2}\right) .
\end{aligned}
$$

Introducing the identification, 


$$
\begin{aligned}
& |0\rangle_{x_{k}}\left\langle\left. 0\right|_{x_{k}}=\hat{x}_{k} \hat{x}_{k}^{\dagger}\right. \\
& |0\rangle_{x_{k}}\left\langle\left. 1\right|_{x_{k}}=\hat{x}_{k}\right. \\
& |1\rangle_{x_{k}}\left\langle\left. 0\right|_{x_{k}}=\hat{x}_{k}^{\dagger}\right. \\
& |1\rangle_{x_{k}}\left\langle\left. 1\right|_{x_{k}}=\hat{x}_{k}^{\dagger} \hat{x}_{k},\right.
\end{aligned}
$$

for the modes $x_{k}=a_{k}, b_{k}, c_{k}(k=1,2, \ldots, N)$, from (21) we obtain a representation of the operator $\mathbb{W}_{k}^{\prime}$ in the Fock space, i.e.,

$$
\hat{\mathbb{H}}_{k}^{\prime}=i \omega_{k} \hat{b}_{k}^{\dagger} \hat{c}_{k}^{\dagger}+\text { H.c., }
$$

where "H.c." means Hermitian conjugate. The operator (23) is known as a two-mode fermion squeezing operator, responsible for creation of fermionic pairs in the framework of the Hawking effect (see e.g., Sec. 5.2 in [15]).

Let us now derive the generator $\mathbb{U}_{k}$, and its Fock space counterpart $\hat{\mathbb{U}}_{k}$, for the entire evolution $U_{k}(\tau)$. By virtue of (18), (15) and (20) we obtain

$U_{k}(\tau)=\mathbb{I}_{k}-i \tau \frac{i \pi}{2}\left(\left|E_{1}\right\rangle_{k}\left\langle\left. E_{4}\right|_{k}-\mid E_{4}\right\rangle_{k}\left\langle\left. E_{1}\right|_{k}\right)+\mathcal{O}\left(\tau^{2}\right)\right.$.

Implementing the identification (22) we obtain the corresponding Fock space generator (Hamiltonian)

$$
\hat{\mathbb{U}}_{k}=\frac{i \pi}{2} \hat{a}_{k} \hat{b}_{k} \hat{b}_{k}^{\dagger} \hat{c}_{k}^{\dagger}+\text { H.c. }
$$

One should note that the operator $\hat{\mathbb{U}}_{k}$ is 4-linear, which should be contrasted with a bilinear structure of the squeezing operator (23) and a trilinear structure of the operator discussed in the context of the Hawking effect in [18]. According to (9) the total Hamiltonian is $\hat{\mathbb{U}}=\sum_{k=1}^{N} \hat{\mathbb{\boxplus}}_{k}$.

Coming back to the global description of the $\mathrm{BH}$ unitary evolution, we would like to draw the reader's attention to a possible interpretation, depicted in Fig. 1. Namely, Fig. 1, in an intuitive way, presents the entire unitary process $U_{k}$ of qubit transfer from a $\mathrm{BH}$ to the outgoing radiation as a composition of the two processes, $U_{k}^{\prime}$ and $U_{k}^{\prime \prime}$, i.e., the Hawking particle pair creation outside the $\mathrm{BH}$ and later particle pair annihilation inside the $\mathrm{BH}$, respectively. A Feynman-like diagram/line depicts the transfer of a qubit in the spirit of a tunneling phenomenon.

Taking into account "reversibility" of unitary processes, the reader could rightfully expect that at some moment of the evolution the "reverse" process of a given one should also occur, and a "reverse diagram" to the one presented in Fig. 1 should also appear. The unitary transformations introduced in Sec. III are actually rotations in eightdimensional complex vector spaces with rotation angles proportional to the time parameter $\tau$. Then, if we start from a vector corresponding to an $a_{k}$ mode and "rotate" it onto a $c_{k}$ mode, and next we stop the evolution, we effectively

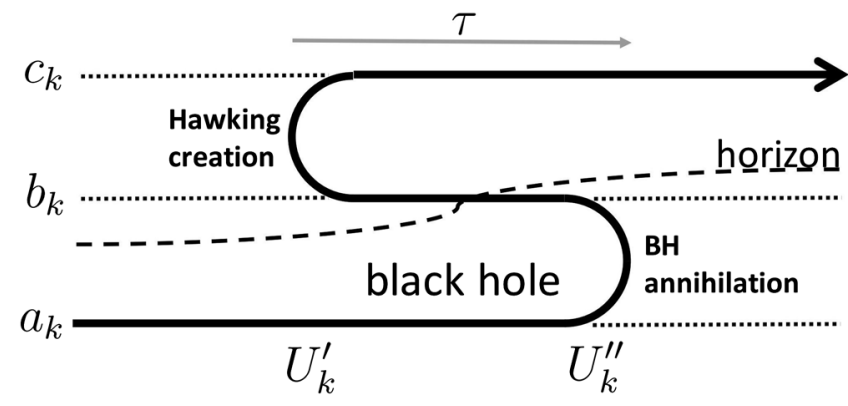

FIG. 1. A Feynman-like diagram/line (the S-shaped thick line with an arrow) depicts the entire qubit transport process $U_{k}$, i.e., a composition of the Hawking particle pair creation $U_{k}^{\prime}$ outside the $\mathrm{BH}$ and of particle pair annihilation $U_{k}^{\prime \prime}$ inside the $\mathrm{BH}$, in the spirit of a tunneling phenomenon.

swap one particle for another. If we instead further continued the evolution we would return to the $a_{k}$ mode back. Therefore, we have to assume that the proposed evolution is only valid within a limited period of time $\tau$, namely $\tau \in[0,1]$. That limitation could be justified by the assumption that the evolution is "effective" rather than "fundamental" in the sense that its temporary form is determined by the current structure of spacetime and the distribution of matter. Then, when $a_{k}$ modes become transformed into $c_{k}$ modes, the situation changes and the evolution in the proposed form switches off. Moreover, one should also note that the whole result is influenced be the initial state of the system, i.e., we start with modes only inside the black hole, while all other modes are in the vacuum state. Such an assumption corresponds to the idealized (model) situation assuming that in the beginning of the evolution we only deal with a (single) black hole (represented by $a_{k}$ modes occupied) and empty spacetime outside the black hole ( $c_{k}$ modes empty).

\section{FINAL REMARKS}

Primarily inspired by a recent paper of Osuga and Page [12], in particular by their Eq. (3.3), essentially the same as Eq. (3.3) in [13] (accidental coincidence of the numbers of the equations!), we have proposed a unitary toy model of $\mathrm{BH}$ evaporation, which is an extension of the model introduced in [14]. By virtue of the construction the model is explicitly unitary and it describes transport of qubits from a $\mathrm{BH}$ to the outgoing radiation. For interpretational simplicity and a more direct relation to particle language, we have decided to formulate our qubit model in terms of fermions. As a by-product of our construction, for possible reference to other models of the Hawking effect and $\mathrm{BH}$ evaporation, besides the global version of the evolution operator, we have determined its infinitesimal form (Hamiltonian). In turn the global evolution, involving the Hawking creation as well as latter annihilation inside the $\mathrm{BH}$, can intuitively be interpreted as a tunneling phenomenon as depicted in Fig. 1. 
[1] S. Chakraborty and K. Lochan, Black holes: Eliminating information or illuminating new physics? Universe 3, 55 (2017).

[2] D. Harlow, Jerusalem lectures on black holes and quantum information, Rev. Mod. Phys. 88, 015002 (2016).

[3] D. Marolf, The black hole information problem: Past, present, and future, Rep. Prog. Phys. 80, 092001 (2017).

[4] J. Polchinski, The black hole information problem, in Proceedings, Theoretical Advanced Study Institute in Elementary Particle Physics: New Frontiers in Fields and Strings (TASI 2015): Boulder, CO, 2015 (World Scientific, Singapore, 2017), pp. 353-397.

[5] S. G. Avery, Qubit models of black hole evaporation, J. High Energy Phys. 01 (2013) 176.

[6] S. B. Giddings, Black holes, quantum information, and unitary evolution, Phys. Rev. D 85, 124063 (2012).

[7] S. B. Giddings, Models for unitary black hole disintegration, Phys. Rev. D 85, 044038 (2012).

[8] S. B. Giddings and Y. Shi, Quantum information transfer and models for black hole mechanics, Phys. Rev. D 87, 064031 (2013).

[9] S.D. Mathur, The information paradox: A pedagogical introduction, Classical Quantum Gravity 26, 224001 (2009).

[10] S. D. Mathur, What exactly is the information paradox? Lect. Notes Phys. 769, 3 (2009).
[11] S. D. Mathur and C. J. Plumberg, Correlations in Hawking radiation and the infall problem, J. High Energy Phys. 09 (2011) 093.

[12] K. Osuga and D. N. Page, Qubit transport model for unitary black hole evaporation without firewalls, Phys. Rev. D 97, 066023 (2018).

[13] A. Almheiri, D. Marolf, J. Polchinski, D. Stanford, and J. Sully, An apologia for firewalls, J. High Energy Phys. 09 (2013) 018.

[14] B. Broda, Unitary toy qubit transport model for black hole evaporation, Eur. Phys. J. C 80, 418 (2020).

[15] R. B. Mann, Black Holes: Thermodynamics, Information, and Firewalls, Springer Briefs in Physics (Springer, New York, 2015).

[16] F. C. Khanna, A. P. C. Malbouisson, J. M. C. Malbouisson, and A. E. Santana, Thermal Quantum Field Theory. Algebraic Aspects and Applications (World Scientific, Hackensack, NJ, 2009).

[17] K. Svozil, Squeezed Fermion States, Phys. Rev. Lett. 65, 3341 (1990).

[18] P. D. Nation and M. P. Blencowe, The trilinear Hamiltonian: A zero dimensional model of Hawking radiation from a quantized source, New J. Phys. 12, 095013 (2010). 